\title{
Occurrence of Pathogenic Aspergillus species in Drinking Water from Restaurants in Kathmandu, Nepal
}

\author{
U Shrestha Khwakhali ${ }^{*}$, JF Meis ${ }^{2,3}$, PE Verweij ${ }^{3,4}$
}

Radboudumc

${ }^{1}$ Department of Microbiology, Amrit Campus, Tribhuvan University, Kathmandu, Nepal

2Department of Medical Microbiology and Infectious Diseases, Canisius Wilhelmina Hospital, Nijmegen, The Netherlands

${ }^{3}$ Centre of Expertise in Mycology Radboudumc/CWZ, Nijmegen, The Netherlands

${ }^{4}$ Department of Medical Microbiology, Radboudumc, Nijmegen, The Netherlands

\section{Introduction}

- Aspergillus is a ubiquitously distributed opportunistic fungus that causes a wide range of infections in both immunocompetent and immunocompromised hosts.

- Aspergillus fumigatus is the global leading cause of invasive aspergillosis associated with high morbidity and mortality.

- A. flavus also causes invasive aspergillosis and is known to produce aflatoxins.

- Aspergillus species and other fungi are accounted as a significant cause of water contamination due to their ability to survive after filtration in distribution networks and during storage even when they have been treated with chlorine.

- The presence of Aspergillus in drinking water can lead to invasive infections, allergy and toxic responses, particularly in immunocompromised patients.

- In this study, we investigated the occurrence of pathogenic Aspergillus species in drinking water from restaurants in Kathmandu, Nepal.

\section{Methods}

- A total of 120 drinking water samples were collected between March to June 2017 from restaurants in the centre of Kathmandu and processed using a membrane filter (MF) technique according to standard methods of American Public Health Association (2005).

- A volume of $100 \mathrm{~mL}$ water was filtered through a sterile membrane filter with $0.45 \mu \mathrm{m}$ pore size and $47 \mathrm{~mm}$ diameter.

- The membranes were placed on Sabouraud dextrose agar plates with chloramphenicol $(50 \mathrm{mg} / \mathrm{L})$ and incubated at $37^{\circ} \mathrm{C}$ for up to 7 days and examined daily for any visible growth of pathogenic fungi.

- Pathogenic Aspergillus species as well as different types of other fungi were enumerated and identified to species complex level by macroscopic and microscopic morphology.

- Microscopy, germ-tube test and biochemical tests were also performed for identification of yeasts.

\section{Results}

- All treated drinking water samples were positive for the growth of pathogenic fungi.

- Aspergillus species were recovered from $63 \%$ of water samples from restaurants but yeasts $(83.7 \%)$ were more predominant than filamentous fungi (16.3\%).

- Total count of Aspergillus species ranged from 1 to 38 colony forming units (cfu)/100 mL, with an average of $5 \mathrm{cfu} / 100 \mathrm{~mL}$.

- The most abundant genera of filamentous fungi identified were Aspergillus (10.2\%) but Fusarium (1.0\%), Penicillium $(0.8 \%)$, Rhizopus (0.4\%), Mucor (0.4\%), Curvularia $(0.3 \%)$ and Trichoderma $(0.2 \%)$ were also isolated.

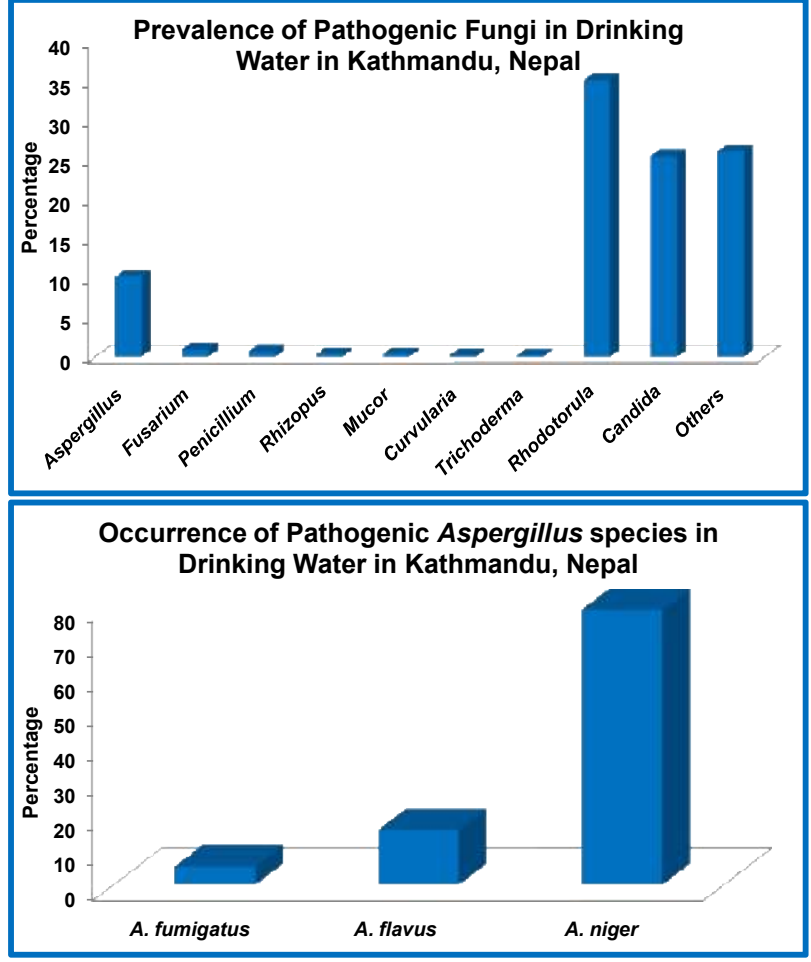

-The genera Rhodotorula (35.1\%) and Candida (25.5\%) were detected in a high frequency.

-Among Aspergillus isolates, A. fumigatus (5.3\%), A. flavus (15.8\%) and $A$. niger $(78.9 \%)$ were recovered from drinking water samples.

\section{Conclusion}

- Pathogenic Aspergillus species were the most frequently isolated filamentous fungi in treated drinking water sources in Kathmandu.

- The occurrence of opportunistic fungal pathogens in drinking water is a potential threat to human health and indicated increased risk of Aspergillus infections.

- Awareness of drinking water quality and water safety and the availability of improved drinking water treatment systems should be emphasized to maintain microbial drinking water safety.

\section{Acknowledgements}

We are grateful to ISHAM for ISHAM2018 attendance grant and Fungal Infection Trust for a travel grant to attend ISHAM 2018. We thank Atmaz Kumar Shrestha and Prashanna Maharjan for their help during water sample collection and filtration.

\footnotetext{
1. Hedayati MT, Pasqualotto AC, Warn PA, Bowyer P, Denning DW. Aspergillus flavus: human Hedayati MT, Pasqualotto AC, Warn PA, Bowyer P, Denning DW. Aspers
pathogen, allergen and mycotoxin producer. Microbiology 2007; 153(Pt 6): 1677-1692.

pathogen, allergen and mycotoxin producer. Microbiology 2007; 153(Pt 6): 1677-1692.
Babič MN, Gunde-Cimerman N, Vargha M, Tischner Z, Magyar D, Veríssimo C, Sabino R, Viegas C, Meyer 2. Babič MN, Gunde-Cimerman N, Vargha M, Tischner Z, Magyar D, Veríssimo C, Sabino R, Viegas C, Meyer
W, Brandão J. Fungal contaminants in drinking water regulation? A tale of ecology, exposure, purification and clinical relevance. Int J Environ Res Public Health 2017; 14: 636.

3. Oliveira HMB, Santos C, Paterson RRM, Gusmão NB, Lima N. Fungi from a groundwater-fed drinking water supply system in Brazil. Int J Environ Res Public Health 2016; 13: 304.

4. Al-gabr HM, Zheng T, Yu X. Occurrence and quantification of fungi and detection of mycotoxigenic fungi in drinking water in Xiamen City, China. Sci Total Environ 2014; 466(467):1103-1111.

5. Hageskal G Gaustad P. Heier BT Skaar I. Occurrence of moulds in drinking water. J Appl Microbiol 2007;

102(3):774-780.

(APHA). Standard method for examination of water and wastewater, 21s edn. APHA, Washington, DC,USA, 2005.
}

Copyright @ 2017 U Shrestha Khwakhali, JF Meis, PE Verweij. E-mail: ushanas@hotmail.com 